\title{
Lithium as a passive tracer probing the rotating solar tachocline turbulence
}

\author{
G. Rüdiger ${ }^{1,3}$ and V. V. Pipin ${ }^{1,2}$ \\ 1 Astrophysikalisches Institut Potsdam, An der Sternwarte 16, 14482 Potsdam, Germany \\ 2 Institute for Solar-Terrestrial Physics, PO Box 4026, Irkutsk 664033, Russia \\ 3 Department of Mathematics, University of Newcastle upon Tyne, NE1 7RU, UK
}

Received 25 February 2000 / Accepted 7 June 2001

\begin{abstract}
The rotational influence on the eddy-diffusivity tensor $D_{i j}$ for anisotropic turbulence fields is considered in order to explain the lithium decay law during the spin-down process of solar-type stars. Rotation proves to be highly effective in the transfer of chemicals through the solar tachocline (beneath the convection zone) which is assumed to contain only turbulence with horizontal motions. The effect is so strong that the tachocline turbulence must not exceed a limit of $\sim 10^{-(3 \ldots 4)}$ of the rms velocity in the convection zone in order to let the lithium survive after Gigayears. Such long depletion times can also be explained by a very small rotational influence upon the eddy-diffusion tensor if it is realized with correlation times shorter than $15 \mathrm{~min}$. It is argued that such slow and/or short-living turbulence beneath the convection zone could hardly drive the solar dynamo. In our theory the diffusion remains small for rapid rotation due to the rotational quenching of the turbulence. In young stellar clusters like Pleiades, there should be a (positive) correlation between rotation rate and lithium abundance, where the fastest stars should have maximal lithium. First inspections of the data seem to confirm this finding.
\end{abstract}

Key words. turbulence - stars: abundances - stars: rotation

\section{Introduction}

According to Duncan (1981), the lithium at the surface of cool main-sequence stars decays exponentially, unlike the stellar spin-down, which follows a power law $t^{-1 / 2}$ (Skumanich 1972). The characteristic decay time is about 1 Gyr. The primordial lithium is destroyed by nuclear reactions at temperatures in excess of $2.6 \times 10^{6} \mathrm{~K}$ (see Michaud \& Charbonneau 1991; Ahrens et al. 1992), i.e. about $40000 \mathrm{~km}$ below the lower edge of the convection zone (Fig. 1). There must be a drift process for the chemicals from the bottom of the convection zone through the solar "tachocline" to the burning domain. The effect must be small, however, in order to allow the existence of lithium in the solar atmosphere even after 4.6 Gyr. The lithium decay time is about $10^{7}$ times the convection zone diffusion time of $\sim 100$ yrs. We are thus looking for a rather small effect which, however, cannot simply be microscopic diffusion (Schatzman \& Maeder 1981; Spruit 1984; Zahn 1989, 1992). In the following, the consequences are presented of a quasilinear mean-field approximation of an anisotropic turbulence field which might be located in the solar "tachocline", which is stably stratified unlike the unstably-stratified solar convection zone. However, to

Send offprint requests to: G. Rüdiger, e-mail: gruediger@aip.de

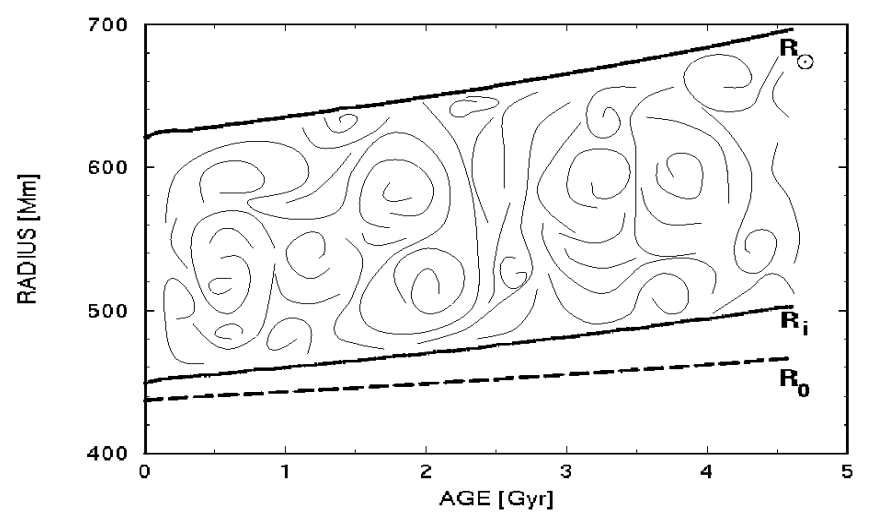

Fig. 1. The outer structure of the Sun during its main-sequence life. $R_{i}$ limits the unstably stratified outer convection zone while $R_{0}$ displays the lithium burning zone with a temperature of 2.6 Mio K. Computed by Kämmerer (2000) with the solar model of Stix \& Skaley (1990).

form reduction factors of $10^{7 \ldots 8}$ for any eddy diffusivity is a nontrivial problem. In other words, the desired eddy diffusion coefficient only exceeds the molecular one by two orders of magnitude (Baglin et al. 1985; Lebreton \& Maeder 1987) and this is not easy to explain (Vincent et al. 1996). 
The transport of a passive scalar is governed by the diffusion equation,

$\frac{\partial \rho C}{\partial t}+\operatorname{div}(\rho C \boldsymbol{u}-\rho D \nabla C)=0$,

with $D$ as the microscopic diffusion coefficient. In the sense of the anelastic approximation we shall always apply the source-free condition of the mass flux, i.e.

$\operatorname{div} \rho \boldsymbol{u}=0$.

If the field $\boldsymbol{u}$ describes a turbulent flow pattern, the fields are split into a mean and a fluctuating part,

$C=\bar{C}+C^{\prime}, \quad \boldsymbol{u}=\overline{\boldsymbol{u}}+\boldsymbol{u}^{\prime}$.

Averaging (1) we get the well-known diffusion equation in the presence of turbulence

$\frac{\partial \rho \bar{C}}{\partial t}+\operatorname{div}\left\{\rho\left\langle C^{\prime} \boldsymbol{u}^{\prime}\right\rangle-\rho D \nabla \bar{C}\right\}=0$.

The influence of a meridional circulation considered by Charbonneau \& Michaud (1991), Zahn (1992) and Chaboyer \& Zahn (1992) is neglected here.

The fluctuations of the chemical concentration in a quasilinear approximation follow the relation

$\frac{\partial \rho C^{\prime}}{\partial t}+\operatorname{div}\left(\rho \bar{C} \boldsymbol{u}^{\prime}-\rho D \nabla C^{\prime}\right)=0$.

From this we have to compute the turbulent concentration flux vector $\left\langle C^{\prime} \boldsymbol{u}^{\prime}\right\rangle$ which must be inserted into (4) in order to find the mean-field diffusion equation. In particular, we are interested in the effect of the basic stellar rotation and its consequences during the stellar spin-down process (see Charbonnel et al. 1992).

\section{The diffusion tensor}

In a corotating frame of reference the equation for the fluctuating part of the velocity field $\boldsymbol{u}^{\prime}$ is

$\frac{\partial \boldsymbol{u}^{\prime}}{\partial t}+2 \boldsymbol{\Omega} \times \boldsymbol{u}^{\prime}+\frac{1}{\rho} \nabla p^{\prime}-\nu_{\mathrm{t}} \Delta \boldsymbol{u}^{\prime}=\boldsymbol{f}^{\prime}$

with $\boldsymbol{f}^{\prime}$ as the random turbulence force and $\nu_{\mathrm{t}}$ as some background eddy viscosity. The desired correlation between concentration fluctuations and velocity fluctuations, $\left\langle C^{\prime} \boldsymbol{u}^{\prime}\right\rangle$, can be found by Fourier transforming (6) using

$\boldsymbol{u}^{\prime}(\boldsymbol{x}, t)=\int \hat{\boldsymbol{u}}(\boldsymbol{k}, \omega) \mathrm{e}^{\mathrm{i}(\boldsymbol{k} \cdot \boldsymbol{x}-\omega t)} \mathrm{d} \boldsymbol{k} \mathrm{d} \omega$.

As usual, the original anisotropic turbulence is assumed to be stationary, homogeneous and anisotropic, i.e.

$$
\begin{aligned}
& \left\langle\hat{u}_{j}^{(0)}(\boldsymbol{k}, \omega) \hat{u}_{j}^{(0)}\left(\boldsymbol{k}^{\prime}, \omega^{\prime}\right)\right\rangle=\delta\left(\omega+\omega^{\prime}\right) \delta\left(\boldsymbol{k}+\boldsymbol{k}^{\prime}\right) \\
& \quad\left\{\frac{E(k, \omega)}{16 \pi k^{2}}\left(\delta_{i j}-k_{i}^{\circ} k_{j}^{\circ}\right)+\frac{3}{16 \pi k^{2}} E_{2}(k, \omega)\left(\delta_{i j}-k_{i}^{\circ} k_{j}^{\circ}\right.\right. \\
& \left.\left.\quad-\left(\boldsymbol{g} \cdot \boldsymbol{k}^{\circ}\right)^{2} \delta_{i j}-g_{i} g_{j}+\left(\boldsymbol{g} \cdot \boldsymbol{k}^{\circ}\right)\left(g_{i} k_{j}^{\circ}+g_{j} k_{i}^{\circ}\right)\right)\right\}
\end{aligned}
$$

(cf. Rüdiger 1989). $E(k, \omega)$ is the isotropic part of the turbulence spectrum, $\boldsymbol{k}^{\circ} \equiv \boldsymbol{k} / k$ and $\boldsymbol{g}$ is the vertical unit vector. The radial turbulence intensity may be denoted by $w^{2}=\left\langle u_{r}^{(0) 2}\right\rangle$ while for the azimuthal turbulence intensity $v^{2}=\left\langle u_{\phi}^{(0) 2}\right\rangle$. An anisotropy parameter $s$ is defined by $v^{2}=s w^{2}$, so that a large $s$ denotes horizontal-type turbulences. It is

$w^{2}=\frac{1}{3} \iint_{0}^{\infty} E(k, \omega) \mathrm{d} k \mathrm{~d} \omega$.

The vertical vector $\boldsymbol{g}$ represents the basic anisotropy, which is described by the spectrum $E_{2}(k, \omega)$ of additive horizontal motions

$v^{2}=\frac{1}{2} \iint_{0}^{\infty} E_{2}(k, \omega) \mathrm{d} k \mathrm{~d} \omega$.

The result for the turbulent concentration-flux vector may be written as an anisotropic diffusion in terms of the mean concentration gradient, i.e.

$\left\langle C^{\prime} u_{i}^{\prime}\right\rangle=-D_{i j} \frac{\partial \bar{C}}{\partial x_{j}}$

(cf. Dolginov \& Silantev 1992). We shall compute in the following the diffusion tensor without rotation and with rotation for the isotropic and anisotropic parts of the turbulence fields.

\subsection{Isotropic turbulence}

The result is very simple without rotation. It follows

$D_{i j}=\frac{1}{3} \iint_{0}^{\infty} \frac{D k^{2}}{\omega^{2}+D^{2} k^{4}} E(k, \omega) \mathrm{d} k \mathrm{~d} \omega \delta_{i j}$,

or just

$D_{i j}=D_{\mathrm{T}} \delta_{i j}$

with the eddy diffusivity $D_{\mathrm{T}} \simeq w^{2} \tau_{\text {corr }}$ formed here only with the vertical turbulence intensity.

More structure results if the turbulence is subject to a basic rotation. The expressions are given here only in the so-called $\tau$-approximation which is very close to the mixing-length approximation (Kitchatinov 1986). We find

$D_{i j}=D_{\mathrm{T}}\left(\Psi_{1} \delta_{i j}+\Psi_{2} \Omega_{i}^{\circ} \Omega_{j}^{\circ}\right)$,

with $\Omega^{\circ}=\Omega / \Omega$ as the vector parallel to the rotation axis. The components of the tensor are

$D_{r r}=D_{\mathrm{T}}\left(\Psi_{1}+\Psi_{2} \cos ^{2} \theta\right)$,

$D_{\theta \theta}=D_{\mathrm{T}}\left(\Psi_{1}+\Psi_{2} \sin ^{2} \theta\right)$,

$D_{r \theta}=D_{\mathrm{T}} \Psi_{2} \sin \theta \cos \theta$

(see Hathaway 1984). The $\Psi_{2}$ ensures a latitudinal transport of the chemical composition. Without rotation 
$\Psi_{1}=1$ and $\Psi_{2}=0$. The rotational quenching functions result as

$\Psi_{1}=\frac{3}{4 \Omega^{* 2}}\left(1+\left(\Omega^{* 2}-1\right) \frac{\arctan \Omega^{*}}{\Omega^{*}}\right)$

and

$\Psi_{2}=\frac{3}{4 \Omega^{* 2}}\left(-3+\left(\Omega^{* 2}+3\right) \frac{\arctan \Omega^{*}}{\Omega^{*}}\right)$

with the Coriolis number $\Omega^{*}=2 \tau_{\text {corr }} \Omega$.

\subsection{Horizontal turbulence}

As usual we consider the temperature profile below the convection zone as stable so that any vertical fluctuations there are strongly suppressed. Any turbulence which possibly exists in the tachocline must be anisotropic. As a model of such an anisotropic turbulence, in this section a strictly horizontal turbulence is considered, i.e., there are, for zero rotation, no vertical fluctuations.

For horizontal turbulence without rotation the diffusion tensor becomes

$D_{i j}=\frac{1}{2} \iint_{0}^{\infty} \frac{D k^{2}}{\omega^{2}+D^{2} k^{4}} E_{2}(k, \omega) \mathrm{d} k \mathrm{~d} \omega\left(\delta_{i j}-g_{i} g_{j}\right)$

without any vertical components. But with rotation and within the $\tau$-approximation we find

$$
\begin{aligned}
D_{i j}= & s D_{\mathrm{T}}\left\{\delta_{i j}\left(\Psi_{3}+3\left(\boldsymbol{g} \cdot \boldsymbol{\Omega}^{\circ}\right)^{2} \Psi_{2}\right)+3 \Psi_{2} \Omega_{i}^{\circ} \Omega_{j}^{\circ}\right. \\
& \left.+\Psi_{4} g_{i} g_{j}+\left(\boldsymbol{g} \cdot \boldsymbol{\Omega}^{\circ}\right) \Psi_{5}\left(g_{i} \Omega_{j}^{\circ}+g_{j} \Omega_{i}^{\circ}\right)\right\},
\end{aligned}
$$

which yields in spherical coordinates the tensor components

$D_{r r}=s D_{\mathrm{T}}\left(\Psi_{3}+\Psi_{4}+\cos ^{2} \theta\left(6 \Psi_{2}+2 \Psi_{5}\right)\right)$,

$D_{\theta \theta}=s D_{\mathrm{T}}\left(\Psi_{3}+3 \Psi_{2}\right)$,

$D_{r \theta}=D_{\theta r}=-s D_{\mathrm{T}} \sin \theta \cos \theta\left(3 \Psi_{2}+\Psi_{5}\right)$.

The amplitude $s$ is introduced as the intensity of the horizontal turbulence in units of the intensity of the isotropic turbulence in the bulk of the convection zone.

The rotational quenching functions result as

$\Psi_{3}=\frac{3}{4 \Omega^{* 2}}\left(7-\left(\Omega^{* 2}+7\right) \frac{\arctan \Omega^{*}}{\Omega^{*}}\right)$,

$\Psi_{4}=\frac{3}{4 \Omega^{* 2}}\left(-\frac{3 \Omega^{* 2}+1}{\Omega^{* 2}+1}+\left(\Omega^{* 2}+1\right) \frac{\arctan \Omega^{*}}{\Omega^{*}}\right)$,

$\Psi_{5}=\frac{3}{4 \Omega^{* 2}}\left(\frac{\Omega^{* 2}+3}{\Omega^{* 2}+1}+\left(\Omega^{* 2}-3\right) \frac{\arctan \Omega^{*}}{\Omega^{*}}\right)$.

Without rotation $\Psi_{3}=-\Psi_{4}=1$ and $\Psi_{5}=0$, only the latitudinal diffusion coefficient $D_{\theta \theta}$ exists. With rotation, the appearance of the vertical diffusion $D_{r r}$ and the offdiagonal components $D_{r \theta}=D_{\theta r}$ can be observed (Fig. 2),

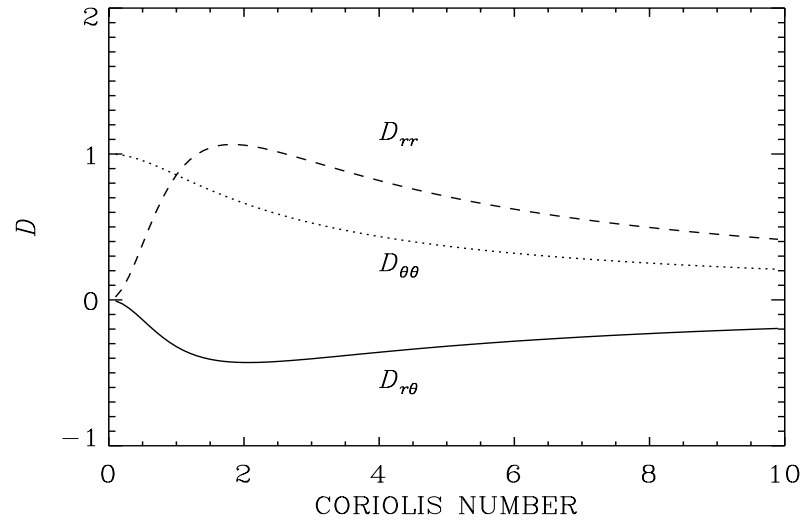

Fig. 2. The diffusion tensor components (22) for midlatitudes $\left(\theta=45^{\circ}\right)$ in their dependence on the Coriolis number $\Omega^{*}$. Note that for fast rotation $\left(\Omega^{*} \geq 1\right)$ all components are rotationally quenched.

which are playing - as we shall see - key roles in the meanfield equation for the large-scale concentration distributions in rotating turbulence fields. One can demonstrate that this equation for all $\Omega^{*}$ remains of the elliptical type.

Note that the influence of rotation is so strong that for $\Omega^{*} \simeq 1$ the vertical diffusion - which is only rotationally created - equals the latitudinal diffusion. Note also that the new diffusion coefficients are strongly $\theta$-dependent, resulting in $\theta$-dependent profiles of the mean concentration (see below).

\section{The model design}

The mean-field diffusion equation without large-scale circulation reads

$$
\begin{aligned}
\rho \frac{\partial \bar{C}}{\partial t}= & \frac{1}{r^{2}} \frac{\partial}{\partial r}\left\{\rho r^{2}\left(D_{r r} \frac{\partial \bar{C}}{\partial r}+\frac{D_{r \theta}}{r} \frac{\partial \bar{C}}{\partial \theta}\right)\right\} \\
& +\frac{1}{r^{2} \sin \theta} \frac{\partial}{\partial \theta}\left\{\rho \sin \theta\left(D_{\theta \theta} \frac{\partial \bar{C}}{\partial \theta}+r D_{r \theta} \frac{\partial \bar{C}}{\partial r}\right)\right\}
\end{aligned}
$$

The boundary conditions are

$\bar{C}=0$

at the lower boundary $\left(x_{0}=0.6\right)$, where the lithium may be destroyed, and

$D_{r r} \frac{\partial \bar{C}}{\partial r}+\frac{D_{r \theta}}{r} \frac{\partial \bar{C}}{\partial \theta}=0$

at the solar surface $(x=1)$ where no radial flux may be allowed.

For the correlation time in the definition of $\Omega^{*}$ a radial profile is used very similar to the radial profile of the turnover time in the mixing-length theory of the solar convection zone, i.e.

$\Omega^{*}=\Omega_{\mathrm{i}}^{*}\left(\frac{x}{x_{\mathrm{i}}}\right)^{7 / 6}\left(\frac{1-x}{1-x_{\mathrm{i}}}\right)^{3 / 2}$

where $\Omega_{\mathrm{i}}^{*}$ is the $\Omega^{*}$-value at $x_{\mathrm{i}}=0.7$. Within the surface layer, $x>0.95$, the Coriolis number $\Omega^{*}$ was put to zero. 


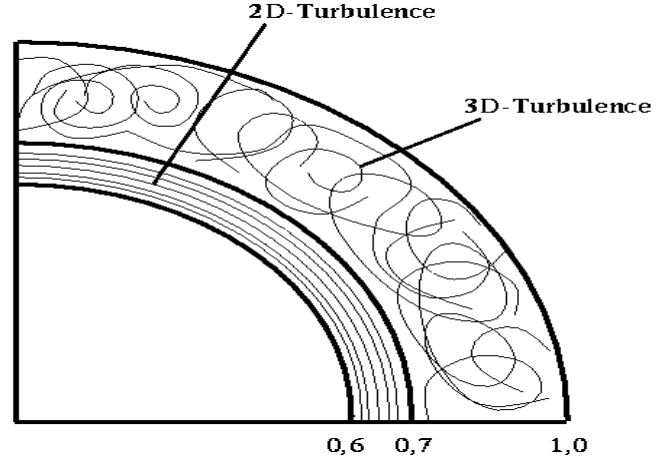

Fig. 3. The overall turbulence model. Below the convection zone with isotropic turbulence there is a stable "tachocline" layer with a horizontal turbulence field. At its bottom lithium is burned.
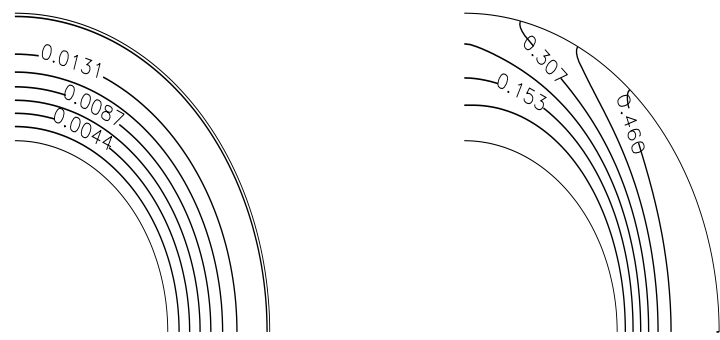

Fig. 4. Isolines of the chemicals without (Left) and with (Right) rotation feedback on the turbulence, $\Omega_{\mathrm{i}}^{*}=6$. In both cases $s=100$. Note that the rotation produces latitudinal profiles in the composition.

Equation (24) is used in dimensionless form, distances measured in units of radius, $R$, and times measured in units of the diffusion time, $\tau_{\text {diff }}=R^{2} / D_{\mathrm{T}}$. We start with a model close to that of Vincent et al. (1996). The diffusivity $D_{\mathrm{T}}$ may be constant in the whole integration region, $x_{0} \leq x \leq 1$. Between the burning zone at $x_{0}$ and the bottom of the convection zone, $x_{\mathrm{i}}$, there is a turbulence field with motions only in the horizontal directions (Fig. 3). Its intensity is given by the parameter $s$. Following Spiegel \& Zahn (1992) we shall refer to the region $x_{0} \leq x \leq x_{\text {i }}$ as the solar "tachocline". The probing of its turbulence with respect to the lithium problem is the main scope of the present study.

The general influence of rotation on the spatial distribution of chemicals inside the star is given in Fig. 4 for both $\Omega_{\mathrm{i}}^{*}=0$ and $\Omega_{\mathrm{i}}^{*}=6$. The rotational influence produces a distinct dependence of the concentration on latitude. Figure 5 shows the decay of chemicals at the surface. The solid line corresponds to non-rotating turbulence for any value of $s$. The same result is obtained even for $s=0$, i.e. without horizontal turbulence. The result of Michaud \& Charbonneau (1991) and Charbonneau (1992) is confirmed in that "horizontal turbulence cannot reduce the effect of a given vertical turbulent diffusion coefficient".

The dashed curve describes the depletion of the chemicals under the action of isotropic but rotating turbulence. Note the rotational slow-down of the chemical depletion. Faster depletion, however, is provided by the extra influ-

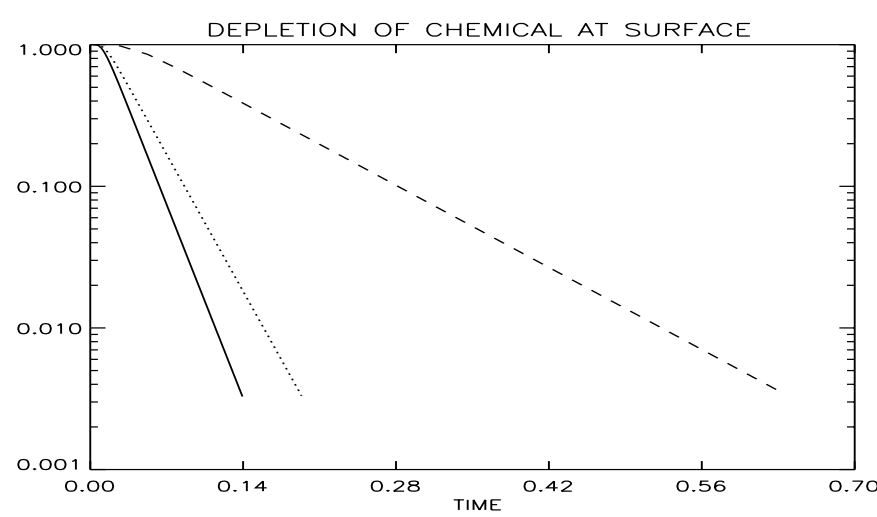

Fig. 5. The decay of chemicals at the surface of the convection zone for isotropic turbulence in the whole computational region, $x_{0}<x<1$. No rotation (solid), with rotation $\left(\Omega_{\mathrm{i}}^{*}=6\right.$, dashed), extra horizontal turbulence in the tachocline beneath the convection zone $\left(\Omega_{\mathrm{i}}^{*}=6, s=100\right.$, dotted).

ence of rotating anisotropic turbulence beneath the convection zone. By this result the influence of the rotation on the turbulence field is reflected. The basic phenomenon is the formation of a latitudinal profile of the concentration as shown in Fig. 4.

We have to take from Fig. 5 that the additional anisotropy due to the basic rotation acts in an unexpected way: rotation suppresses the mixing of isotropic turbulence but, on the other hand, it enables a horizontal turbulence (which without rotation would not be active) to accelerate the vertical mixing. The rotation in the mixing process which is demonstrated in Fig. 5 has a very complex character: it both suppresses and deforms the turbulence. The interplay between both procedures yields the resulting effect. We shall see that for slow rotation enhanced diffusion dominates while for fast rotation the diffusion reduces the suppression of turbulence. As a result for a given rotation rate a maximal diffusion rate exists between slow and fast rotation.

\section{Lithium depletion and the dynamo problem}

Now the turbulence in the convection zone is considered as isotropic turbulence under the influence of rotation described in Sect. 2.1. Below the convection zone the turbulence may be so anisotropic that vertical motions do not exist. The basic rotation, however, produces off-diagonal components in the diffusivity tensor as described in Sect. 2.2. Thus we have 2 free parameters for the tachocline turbulence: intensity $s$ of the horizontal motion and correlation time $\tau_{\text {corr }}^{(2)}$ of the eddies. For $s \rightarrow 0$ the turbulence completely disappears and for $\tau_{\text {corr }}^{(2)} \rightarrow 0$ the rotational influence disappears. In both limits the decay time of the chemicals at the solar surface must become infinite.

More information is given in Fig. 6, where the radial dependencies of the concentration profiles inside the star are given for three different latitudes and two different horizontal intensities. The diffusion is now much slower 


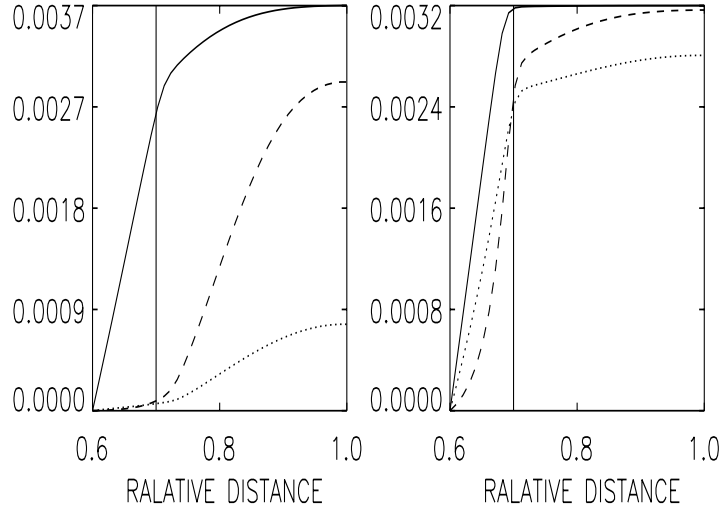

Fig. 6. Radial profiles of the chemical concentration along the equator (solid), mid-latitudes (dashed) and the poles (dotted) for $s=1$ (Left) and $s=0.01$ (Right). Only for small horizontal intensities does the pole-equator difference become rather small. The time-dependence is now very weak, $\Omega_{\mathrm{i}}^{*}=6$ at the base of the convection zone and also within the tachocline.

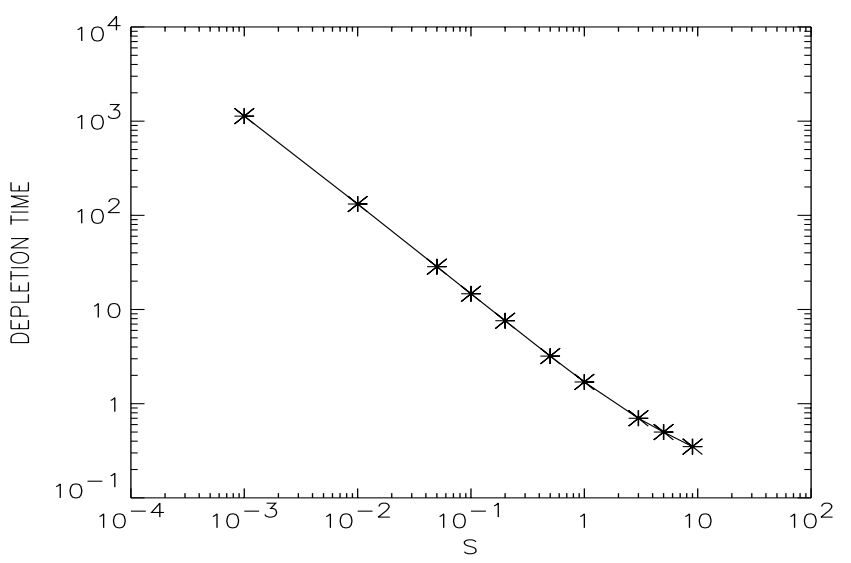

Fig. 7. Depletion time in units of the diffusion time. For weak horizontal turbulence intensities it approximately varies with $1 / s . \Omega_{\mathrm{i}}^{*}=6$ at the base of the convection zone and also within the tachocline.

than with isotropic turbulence in the whole computational space, as in Fig. 5. The differential concentrations are produced in the tachocline and are directly imprinted at the surface and are therefore not screened by the convective pattern within the convection zone. This is the same situation as for differential rotation but different from turbulent heat transport (cf. Stix 1981). The time-dependence of the surface differences of the concentration is so weak that our snapshots are always characteristic the situation. In Fig. 7 the decay time of an initially uniform concentration is plotted for various horizontal turbulence intensities in the tachocline. For $s=0$, of course, there is no depletion, but the depletion time is strikingly short for nearly homogeneous turbulence fields with (say) $s=1$. The correlation time profile and the basic rotation are taken from a solar model by Stix \& Skaley with $\Omega^{*}=6$ at the base of the convection zone. We find that rotation is highly effective in transporting the chemicals to the burning zone at $x_{0}$. A scaling such as

$\tau_{\mathrm{dec}} \simeq \tau_{\mathrm{diff}} / s$

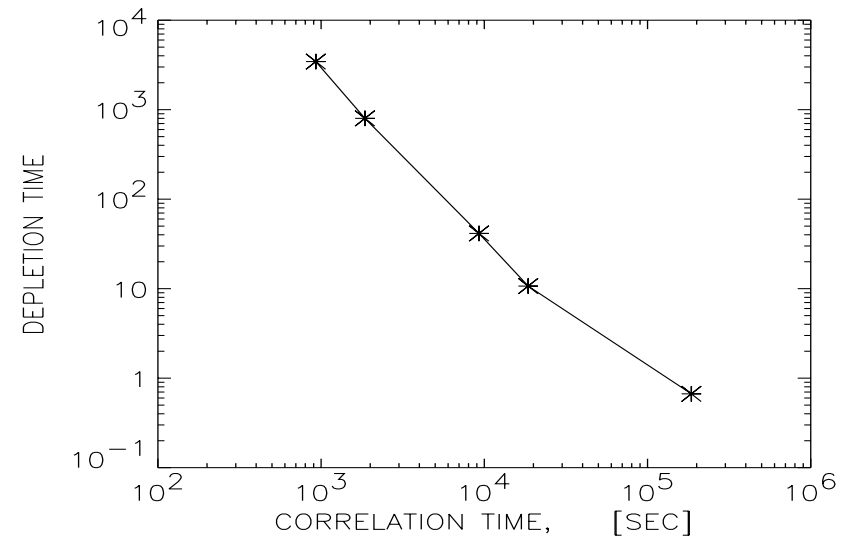

Fig. 8. Depletion time $\tau_{\text {dec }}$ (in units of the diffusion time) for horizontal turbulence intensities with $s=1$ strongly depends on the correlation time of the tachocline turbulence, taken here for a rotation period of 25 days.

is found compatible with the idea that the diffusion of horizontal turbulence under the influenc of rotation with $\Omega^{*} \simeq 1$ can be considered approximately as diffusion of isotropic turbulence. In reality, both the characteristic times differ by 7 orders of magnitude. Therefore, the horizontal rms velocity of the tachocline turbulence must not exceed $10^{-(3 \ldots 4)}$ of the convection zone turbulence. That there is still lithium at the solar surface is only compatible here with the given concept of rotating turbulence with very small $s$ of the tachocline turbulence. Consequently, the horizontal turbulence intensities must be very low, i.e. of the order of $1 \mathrm{~cm} / \mathrm{s}$. There is certainly no chance of maintaining magnetic fields of the order of kGauss by such a slow flow.

But there is also the possibility that the tachocline turbulence has a rather short correlation time, e.g. similar to that of granulation at the top of the convection zone. Then the Coriolis number, $\Omega^{*}$, is smaller than unity and the rotational influence can only be very small. For a given intensity $(s=1)$ the resulting depletion times are shown in Fig. 8. We find a relation $\tau_{\text {dec }} / \tau_{\text {diff }} \simeq 10^{9} / \tau_{\text {corr }}^{2}$ with $\tau_{\text {corr }}$ in seconds. Similarly, for $s=0.01$ we find

$\tau_{\text {dec }} \simeq \frac{10^{14}}{\tau_{\text {corr }}^{2}} \tau_{\text {diff }}$

from our simulation. In the latter case with $\tau_{\text {corr }} \simeq$ $10^{3 \ldots 4} \mathrm{~s}$ the lithium decay time will approach the order of $10^{9}$ yrs. The corresponding Coriolis number is always smaller than 0.05. Again, with such a small value there is no hope of forming an effective $\alpha$-effect for an appropriate dynamo process. In this approach, we find a close relation between the lithium problem and the theory of the solar dynamo. Within this framework, the observations do not favour the existence of an overshoot dynamo below the bottom of the convection zone.

\section{Rotation rate variations}

For a given stellar model the basic rotation also forms a free parameter which can be varied. One can vary the 


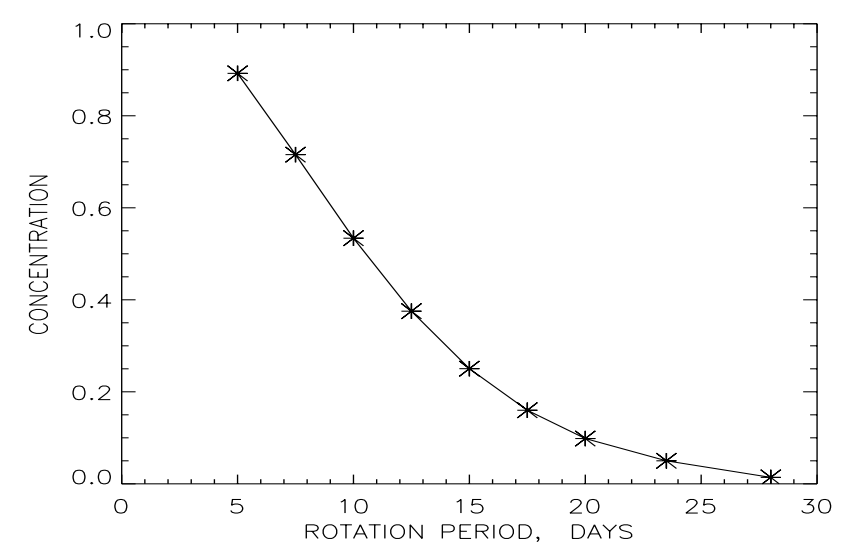

Fig. 9. The general situation for young stellar clusters: the same turbulence model with $s=0.01$ but the angular velocity varied. The surface concentration at the equator is shown after 100 diffusion times. Due to the rotational quenching of the eddy diffusivity, the fast rotators possess the highest surface concentration of chemicals.

basic rotation rate such as it varies in a young stellar cluster for all stars with the same age. The question is how the lithium varies. The same turbulence model can be used within the sample. We take $s=0.01$ and $\tau_{\text {corr }}^{(2)}=$ 12 days. Figure 9 shows the results. Due to the rotational quenching of the eddy diffusivity (see Boubnov \& Golitsin 1995), the faster the rotation the more lithium remains at the surface. The rotational quenching of the eddy diffusivity dominates the effect of the large latitudinal gradients of the concentration. The slower rotators are thus more effective in mixing the chemicals downwards. The plot, however, does not display that for still slower rotation, in the limit for $\Omega^{*}<1$, the mixing again becomes slower and slower and therefore the decay of the primordial lithium, too. The observation of the lithium-rotation correlation in young stellar clusters seems to confirm our prediction.

\section{Conclusions}

Rotation easily produces off-diagonal elements and other new terms in the diffusion tensor. We have shown with our computations that under the rotational influence, even a horizontal turbulence field will be very effective in transporting chemical components in the vertical direction. Two main consequences of this finding have been formulated, i.e.

- even a strictly horizontal turbulence in the solar tachocline must be very weak (or very short-lived) to ensure the observed slow diffusion of lithium downward to the 2.6 Mio K layer where it burns;
- for G-stars in young stellar clusters a correlation should exist between the lithium abundance and the actual rotation rate. The faster the rotation, the stronger the eddy diffusivity is quenched and the higher the lithium concentration in the convection zone must be.

Our concept also provides statements for the long-term evolution of the lithium abundance under the influence of the general spin-down of solar-type stars. To this end a time-dependent code (which is in preparation) must simultaneously include both the time and depth-dependent effects of lithium burning and the decay law of the rotation rate with time.

Acknowledgements. V. V. Pipin acknowledges the kind support by the Deutsche Forschungsgemeinschaft (436 RUS 113/255). The referee, J. P. Zahn, is acknowledged for many valuable suggestions concerning the concept of the paper.

\section{References}

Ahrens, B., Stix, M., \& Thorn, M. 1992, A\&A, 264, 673

Baglin, A., Morel, P. J., \& Schatzman, E. 1985, A\&A, 149, 309

Boubnov, B. M., \& Golitsin, G. S. 1995, Convection in Rotating Fluids (Kluwer Academic Publishers, Dordrecht, London)

Chaboyer, B., \& Zahn, J.-P. 1992, A\&A, 253, 173

Charbonneau, P. 1992, A\&A, 259, 134

Charbonneau, P., \& Michaud, G. 1991, ApJ, 370, 693

Charbonnel, C., Vauclair, S., \& Zahn, J.-P. 1992, A\&A, 255, 191

Dolginov, A. Z., \& Silantev, N. A. 1992, GAFD, 63, 139

Duncan, D. K. 1981, ApJ, 248, 651

Hathaway, D. H. 1984, ApJ, 276, 316

Kämmerer, S. 2000, Ph.D. Thesis, Potsdam

Kitchatinov, L. L. 1986, GAFD, 35, 93

Lebreton, Y., \& Maeder, A. 1987, A\&A, 175, 99

Michaud, G. J., \& Charbonneau, P. 1991, Space Sci. Rev., 57, 1

Rüdiger, G. 1989, Differential Rotation and Stellar Convection (Gordon \& Breach Sc. Pub., New York)

Schatzman, E., \& Maeder, A. 1981, A\&A, 96, 1

Skumanich, A. 1972, ApJ, 171, 565

Spiegel, E. A., \& Zahn, J.-P. 1992, A\&A, 265, 106

Spruit, H. C. 1984, Mixing in the solar interior, in Proc. of the Fourth European Meeting on Solar Physics, The Hydrodynamics of the Sun (Noordwijk), 21

Stix, M. 1981, A\&A, 93, 339

Stix, M., \& Skaley, D. 1990, A\&A, 232, 234

Vincent, A., Michaud, G., \& Meneguzzi, M. 1996, Phys. Fluids, 8, 1312

Zahn, J.-P. 1989, Theory of transport processes, in IAU Coll. 121, ed. G. Berthomieu, \& M. Cribier, Inside the Sun (Kluwer), 425

Zahn, J.-P. 1992, A\&A, 265, 115 